Gut 2006;55:733-741. doi: 10.1136/gut.2005.076612

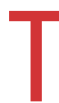

he diagnosis of Crohn's disease is established through an assessment of the clinical presentation with confirmatory evidence from radiographic, endoscopic, and pathological findings. As there is no known cure, and the condition runs a relapsing course, patients are recurrently reinvestigated. Although ultimately curable by colectomy, many patients with ulcerative colitis (UC) are managed medically for years, and require investigations to define the extent of disease, to re-examine diagnosis validity as clinical findings change, and to facilitate dysplasia surveillance. Imaging studies in these diseases serve to establish the primary diagnosis and to provide information for guiding the management of patients with known disease. ${ }^{1}$

Conventional investigations in patients with suspected or proven inflammatory bowel disease include colonoscopy, ileal intubation, and small bowel follow through (SBFT) or small bowel enteroclysis (SBE). It is recognised that these diagnostic modalities may not be uniformly confirmatory. Complete colonoscopy is not always possible due to technical difficulties, poor preparation, or patient intolerance. While the rate of ileal intubation by experienced endoscopists is reported to be $74 \%$, that rate exceeds $90 \%$ when intubation is deemed necessary. ${ }^{2}$ A recent study reports that the rate of ileal intubation is low in the presence of an endoscopically normal colon, even when symptoms suggestive of inflammatory bowel disease are present. ${ }^{3}$ of the 20638 patients who underwent colonoscopy in the setting of an endoscopically normal colon for the indication of abdominal pain, diarrhoea, or anaemia, ileal intubation was performed only $18 \%$ of the time.

SBFT is considered a reliable approach to imaging the small bowel in inflammatory bowel disease, provided that it is a dedicated study, incorporating fluoroscopy with manual manipulation. ${ }^{4}$ The sensitivity and specificity of SBFT in terminal ileal disease is reported in the range of $85-95 \%$ and $89-94 \%$, respectively, but these parameters are highly dependent on the experience of the radiologist. ${ }^{56}$ Although some radiologists favour $\mathrm{SBE}^{78}$ using a nasally placed enteric tube, SBFT can be at least as sensitive as SBE and is preferred by patients as no nasal intubation is necessary. ${ }^{9}$

In Europe, more so than in North America, transabdominal ultrasound (US) has become a standard approach to abdominal imaging for Crohn's disease. US will be reviewed below.

Early diagnosis and sometimes reassessing disease extent or activity of inflammatory bowel disease can be challenging. Patients with established inflammatory bowel disease typically undergo many investigations over a lifetime. Novel imaging studies are emerging which offer advantages in the diagnosis, follow up, and management of Crohn's disease and UC. This article addresses those advances. Table 1 contrasts the various imaging techniques for small bowel Crohn's disease, the intestinal site that engenders the most controversy regarding imaging.

\title{
WIRELESS CAPSULE ENDOSCOPY
}

See end of article for authors' affiliations

Correspondence to: Dr C N Bernstein, 804F-715 McDermot Avenue, University of Manitoba, Winnipeg, Manitoba, Canada R3E3P4; cbernst@cc.umanitoba.ca
Approximately 40\% of Crohn's disease patients have disease involving both the small bowel and colon. Another one third has disease confined to the small bowel, primarily the ileum. ${ }^{10}$ The small bowel is notoriously difficult to evaluate. Wireless capsule endoscopy (WCE), initially developed for small bowel investigations in patients with occult bleeding, has been studied in small bowel Crohn's disease. Over 10000 WCE examinations have been performed for a variety of indications worldwide with a complication rate of $0.75 \% .{ }^{11}$ WCE enables a painless and radiation free examination of much of the small bowel in an unsedated patient. The patient swallows a capsule which contains a videochip, transmitter, and battery; video images are transmitted to a portable device and later downloaded to a computer. The images are usually interpreted by a gastroenterologist. Small bowel transit time is approximately four hours; the capsule is passed through the stool in 24-48 hours. Equipment costs range form \$US20 000 to \$30000. ${ }^{12}$ Interpretation time varies from 30 minutes to two hours, depending on experience, but is typically one hour. ${ }^{12}$ 
Table 1 Imaging tests in small bowel Crohn's disease

\begin{tabular}{lllll}
\hline Modality & Fistula & Abscess & Stricture & Inflammation \\
\hline SBFT or SBE & ++ & - & ++ & ++ \\
Colonoscopy & + & - & ++ & ++ \\
MRI & ++ & ++ & ++ & ++ \\
WCE & - & - & + & ++ \\
US & + & + & + & ++ \\
CT enteroclysis & ++ & ++ & ++ & ++ \\
Double balloon enteroscopy & - & - & + & \\
\hline SBFT, small bowel follow through; SBE, small bowel enteroclysis; MRI, magnetic resonance imaging; WCE, wireless \\
capsule endoscopy; US, ultrasound; CT, computed tomography. \\
- , not reliable; +, somewhat or potentially useful; ++, generally reliable.
\end{tabular}

WCE is emerging as a modality, superior to other conventional studies. The first published articles on the use of WCE in suspected small bowel diseases appeared in 2002. Costamagna and colleagues ${ }^{13}$ compared WCE to SBFT in 20 patients who underwent both barium $x$ ray and WCE. The barium study was deemed diagnostic in four of 20 patients; WCE was diagnostic in nine of 20. Among the first studies dealing exclusively with inflammatory bowel disease was a paper by Fireman and colleagues ${ }^{14}$ who reported a $71 \%$ yield in diagnosing small bowel Crohn's disease by WCE. Twelve of 17 patients with a normal colonoscopy and small bowel $x$ ray, but with a high clinical suspicion of having Crohn's disease, were found to have lesions regarded as consistent with the condition. ${ }^{15}$ These lesions were mucosal erosions, ulcers, and strictures, interpreted as suggestive of Crohn's disease. What actually constitutes Crohn's disease remains debatable. Ge and colleagues ${ }^{16}$ prospectively reviewed 20 patients with suspected Crohn's disease and a normal SBFT. Thirteen of 20 patients $(65 \%)$ were found to have small bowel lesions, including erosions, aphthous ulcers, nodularity, and ulcerated stenosis. Two other studies likewise reported that WCE yielded information supporting the diagnosis of Crohn's disease in suspected patients with a normal colonoscopy and small bowel $x$ ray in nine of 21 patients in one study ${ }^{17}$ and in 30 of 50 patients in another. ${ }^{18}$

Liangpunsakul and colleagues ${ }^{19}$ assessed 40 patients with a negative SBE and colonoscopy. WCE detected small bowel ulcers, suggestive of Crohn's disease in three patients; SBE detected no ulcerations. A prospective study by Voderholzer and colleagues ${ }^{20}$ compared WCE with computerised tomography (CT) enteroclysis in 22 patients, eight of whom had Crohn's disease. While WCE identified small bowel lesions in seven of eight patients, CT enteroclysis was abnormal in five of eight patients. In another prospective study by the same author in 2005, the frequency of small intestinal Crohn's disease detected by WCE was double that found on CT enteroclysis $(p=0.004) .{ }^{21}$ The gain was mainly due to detection of small mucosal lesions such as erosions and aphthous ulcerations in the jejunum and proximal ileum. There was no significant difference in the detection of lesions in the terminal ileum. Eliakim and colleagues ${ }^{22}{ }^{23}$ compared the diagnostic yield of WCE and CT enteroclysis in 35 patients with suspected Crohn's disease. WCE identified erosions, aphthous ulcers, erythema, and lymphoid hyperplasia in $77 \%$ of patients. SBFT and CT enteroclysis identified abnormalities of wall thickening, nodularity of the terminal ileum, and ulcerations in $50 \%$ of patients. It remains to be determined to what degree these mucosal changes can be deemed to represent Crohn's disease, but undoubtedly for many, true Crohn's disease lesions are uncovered with WCE that had not previously been recognised with SBFT, SBE, or CT enteroclysis.

WCE appears to be safe in the paediatric population. Arguelles-Arias and colleagues ${ }^{24}$ found that WCE was well tolerated in adolescents aged 12-16 years and identified lesions compatible with Crohn's disease in 58\% of patients with a previous negative gastroscopy, colonoscopy, and small bowel $x$ ray. Likewise, Barkay demonstrated good tolerance in adolescents being evaluated for enteropathy..$^{25}$ Because of the difficulty in swallowing the capsule, it may need to be placed endoscopically in very young children.

WCE is more sensitive than conventional modalities. It is easy to perform and is well tolerated by patients. The question is whether WCE should become an initial investigation in the diagnosis of or in the re-evaluation of Crohn's disease. Despite its attributes, WCE has disadvantages. It is expensive. Lesions can be missed due to poor bowel preparation, rapid or delayed small bowel transit time, or orientation of the camera away from a lesion. WCE does not allow biopsy or therapeutic intervention. The ability to determine the exact locations of small bowel lesions is limited other than when they are in the proximal duodenum or the terminal ileum. As WCE is a video representation of the intestinal mucosa, it does not assess the extraluminal manifestations of the disease. The absence of a clear definition of normal versus Crohn's disease remains problematic. WCE is more sensitive than small bowel $x$ ray studies but specificity and positive predictive values are not established. ${ }^{15}$ The significance of red spots, erosions, and mucosal breaks, described in the literature, is not known. Fourteen per cent of asymptomatic patients have aphthous ulcers on WCE. ${ }^{15}$ Sixty eight to seventy one per cent of patients receiving anti-inflammatory medications have small bowel erosions. ${ }^{26}{ }^{27}$ Prospective studies with adequate controls and predetermined end points are necessary to define normality and validate the use of the capsule in early or mild disease.

The capsule can become lodged in a stricture, necessitating surgery. In the largest reported series of 900 patients, evaluated primarily for obscure bleeding, capsule retention occurred in $0.7 \%$ of patients. ${ }^{28}{ }^{29}$ Current practice is that a small bowel $x$ ray is obtained prior to WCE evaluation in Crohn's disease. Alternatively, the evolution of the patency capsule may negate this precautionary measure. Recently developed in Israel, the patency capsule is not yet widely available. If retained in the gastrointestinal tract the patency capsule disintegrates; its fragments are theoretically able to pass through strictures. Boivin and colleagues ${ }^{30}$ studied the 

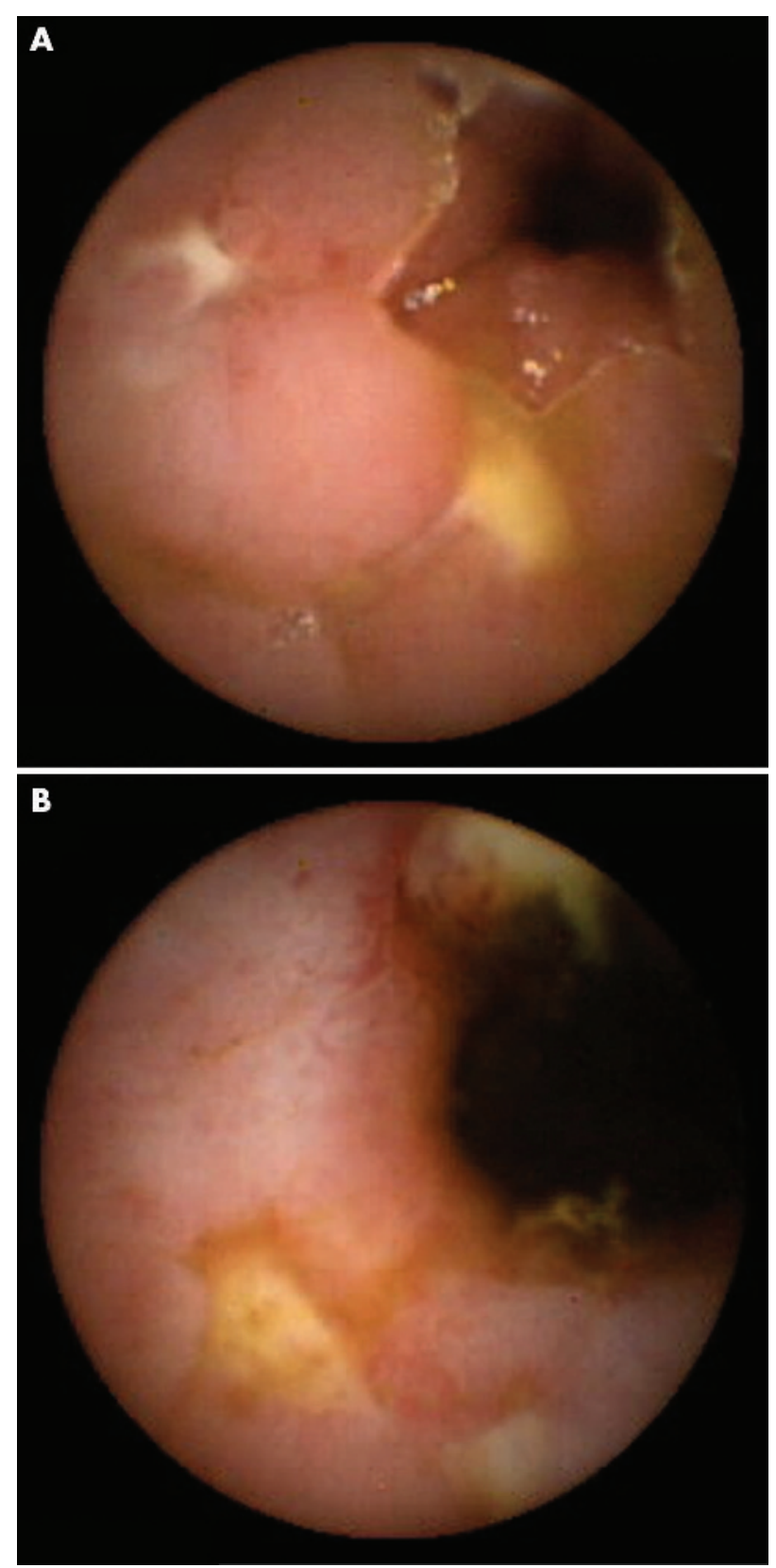

Figure 1 (A, B) Wireless capsule endoscopy (WCE) images of a 47 year old female with a longstanding history of abdominal pain, previously labelled as irritable bowel syndrome and a history of depression. One year previously she presented to another hospital with a possible small bowel obstruction. A small bowel follow through queried possible terminal ileal disease. A subsequent computed tomography scan of the abdomen was normal. She then had a normal colonoscopy although the terminal ileum could not be intubated. Two months later she underwent repeat small bowel follow through which was normal. Because of recurrent abdominal pain and diarrhoea she underwent WCE. The images show mildly stenosed small bowel with ulcers, consistent with Crohn's disease.

patency capsule in 22 patients with suspected small bowel strictures or radiographic small bowel strictures. Sixteen patients passed an intact capsule despite radiological stenosis. Disintegration of the capsule occurred in five patients. In one patient the capsule had to be removed surgically. Although promising, the role of the patency capsule is not fully defined. Occasionally lodging of a capsule
Wireless capsule endoscopy

- WCE is more sensitive than conventional imaging modalities.

- Specificity and positive predictive values for WCE have not been established.

- Because of the costs relative to conventional imaging and the inability to take tissue samples, WCE is unlikely to become the primary modality to initiate a diagnosis of $C D$ in routine cases.

- WCE can diagnose small bowel disease in some instances when CD has been considered and conventional tests are negative.

- WCE is not an essential tool to diagnose CD when extent is known or when documenting the extent of disease will not affect management.

in a stricture in Crohn's disease may help delineate an important site of disease that will likely require surgical intervention.

Based on cost effectiveness, funding issues, and the inability to take tissue samples, it is unlikely that WCE will become the primary imaging modality used to initiate a diagnosis of Crohn's disease or define its extent at possible relapse. WCE can diagnose small bowel disease in some instances where the disease is considered and conventional imaging is negative. It is not a necessary tool to diagnose Crohn's disease when the extent is known or when the extent of the disease will not affect management. If the disease is known and management will be affected by further defining recurrent disease WCE can be of value.

Figure 1 is an example of WCE diagnosing Crohn's disease in a patient where other modalities failed to clinch the diagnosis.

\section{MAGNETIC RESONANCE IMAGING}

Magnetic resonance imaging (MRI) is having an increasing diagnostic impact on patients suffering from inflammatory bowel disease. Its attributes include high soft tissue contrast, multiplanar capabilities, and the use of non-ionising radiation. It can provide information about extraintestinal intraabdominal structures and is functional as well as anatomical. MRI has been shown to be superior to CT scan and fistulography in assessing perineal complications of Crohn's disease as well as fistula and sinus tracts. ${ }^{31}$ Not only does MRI avoid substantial radiation to the pelvis when compared with small bowel $x$ ray or CT, some fistulas escape detection or are diagnosed as inflammatory streaking on CT if air or barium do not enter the tract. ${ }^{32}{ }^{33}$ Furthermore, MRI, because of direct coronal and sagittal imaging, may overcome CT difficulties in identifying the levator ani, a landmark for distinguishing supralevator abscesses. ${ }^{31}$

Historically, intestinal assessment with MRI was suboptimal as slow sequences caused respiratory and peristaltic artefacts. Faster Tl pulse sequences are now routinely used such as fast multiplanar spoiled gradient recalled sequences. Faster T2 signal shot fast spin echo (SSFSE) sequences allow both breathhold and breathing independent images with no physiological artefacts. Luminal distension with oral contrast material provides assessment of the bowel wall and its thickness and regularity. Some centres now use MR enteroclysis, meaning a nasojejunal tube is passed just below the duodenaljenujal flexure and oral contrast is then delivered. Enteroclysis allows better luminal distension but causes patient discomfort. 

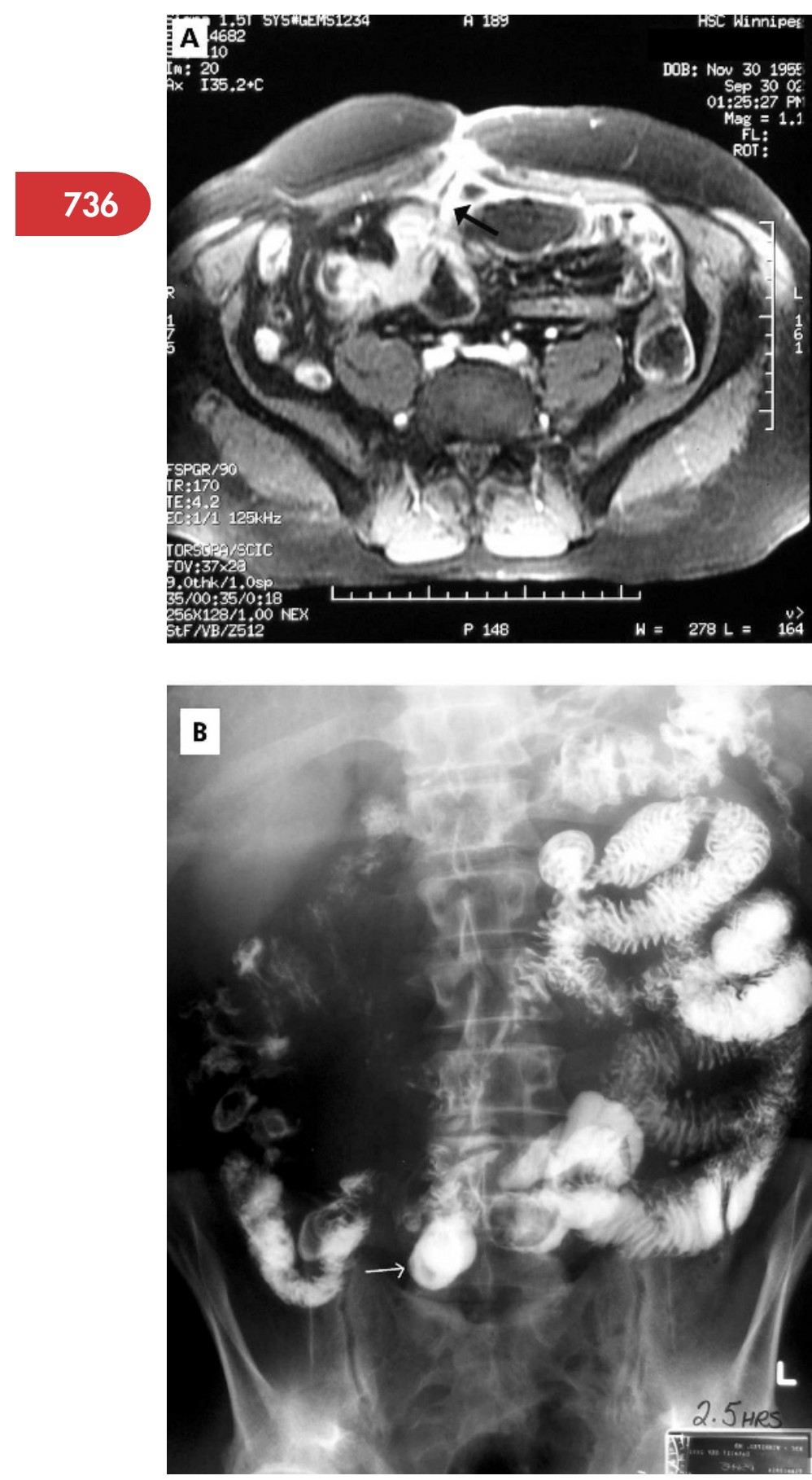

Figure 2 (A) An enterocutaneous fistula that is robustly visualised on axial T1 fast multiplanar spoiled gradient recalled sequence imaging. (B) There is a hint of a fistula on the small bowel follow through but it is much less clearly identified (arrow). Reprinted with permission from the American Journal of Gastroenterology 2005. ${ }^{39}$

MRI has been compared with other imaging modalities in Crohn's disease. MRI using the oral contrast, polyethylene glycol, was compared with ileocolonoscopy in 26 paediatric patients with active Crohn's disease and 49 others presenting to a gastroenterology clinic. For Crohn's disease MRI had a sensitivity of $84 \%$ and a specificity of $100 \%{ }^{34}$

MRI with oral contrast was compared with SBE in a study of 21 patients with Crohn's disease. MRI identified all pathology that was identified on SBE and additional information in six of 21 patients..$^{35}$ The contrast used was 3 litres of pineapple juice mixed with methylcellulose. Overall SBE and MRI with oral contrast revealed similar findings with regard to disease localisation and complications. Three of 21 patients had fistulas on SBE and all three were evident on MRI. In four Crohn's disease patients whose disease extent was either confirmed histologically or surgically, the authors reported markedly higher rates of fistula identification with MRI than with SBE. In four additional patients bowel thickening or contrast enhancement of surrounding fat was identified only by MRI and in one patient salpingitis was identified. Another study of MRI enteroclysis versus SBE in 84 selected Crohn's patients, whose disease was either confirmed surgically or histologically, reported markedly higher rates of fistula identification with MRI than with SBE. ${ }^{36}$ In a study of 25 patients with Crohn's disease and low disease activity, 13 patients had inflammation identified proximal to the terminal ileum by MRI enteroclysis whereas this was identified in only four by SBE. ${ }^{37}$ In the same study, MRI enteroclysis revealed terminal ileum inflammation in 16 of 18 patients with endoscopically and histologically proven inflammation at that site. While SBE revealed terminal ileum inflammation in only 13 of 18 , this was not significantly different from MRI. It has been shown with SBE that there may be washout in the terminal ileum by the time barium reaches this site. ${ }^{9}$ MRI enteroclysis has also been undertaken using oral iron particles as oral contrast and adequately identified Crohn's complications. ${ }^{38}$

A more recent prospective study by Bernstein et al in 2005 compared the utility of MRI with SBFT in the assessment of known Crohn's disease. ${ }^{39}$ Thirty subjects, aged over 18 years underwent both SBFT and MRI. SBFT was performed by a single radiologist, and within four weeks MRI, using $2 \%$ barium sulphate, was performed by another radiologist who was blinded to the SBFT results. After enhanced Tl weighted images and SSFSE, intravenous glucagon and gadolinium were given. Fast multiplanar spoiled gradient recalled Tl coronal sequences were obtained followed by abdominal and pelvic axial images. MRI and SBFT were compared for the extent of disease, presence of complications, and identification of extraintestinal disease. Ten of 30 studies were normal by both modalities and eight showed a similar extent of Crohn's disease. SBFT revealed additional information in four patients, including a stricture in one and ileosigmoid fistulas in two. MRI provided enhanced information in eight patients, including identifying active inflammation in strictured areas based on wall enhancement patterns, vasa recta changes, and lymphadenopathy. The authors concluded that MRI should be the modality of choice in assessing for recurrent Crohn's disease in expert centres, but that SBFT remained a viable screening tool, particularly in centres without MRI expertise.

The most important advantage of MRI over small bowel $x$ ray is the ability to differentiate active inflammation from fibrosis in a thickened segment of bowel..$^{40}$ This distinction has management implications in that the finding of a fibrotic narrowing favours earlier surgery while inflammation would favour a more aggressive medical approach. Koh and colleagues $^{41}$ have suggested that the layered pattern of enhancement on Tl with gadolinium is highly specific for active disease. Similarly, the bright bowel wall due to increased signal of water on T2 SSFSE sequences suggests disease activity.

An important consideration in the adoption of MRI pertains to cost. The incremental cost effectiveness of MRI 
enteroclysis versus SBE was 1595 Euro per additional correctly diagnosed patients. ${ }^{42}$ These data were generated with a decision analytic model relying on one centre's data which included sensitivity for enteric fistula by SBE of $17 \%$. Further analysis is necessary.

MRI is a potentially ideal imaging modality for patients with Crohn's disease who require repeated cross sectional imaging. Lack of ionising radiation, particularly in patients of reproductive age, is appealing. It is safe in patients with renal failure and iodine allergies. It is helpful in patients with equivocal findings by other imaging modalities. The greatest advantage is the ability to identify active inflammation and distinguish whether obstructions are from fibrotic strictures, adhesions, or active inflammation. In fact, minimally invasive (no intravenous contrast required) T2 imaging can be utilised to this end in patients presenting with small bowel obstructions on plain $x$ ray. These patients may not even require oral contrast as their own gastric contents act as good oral contrast on $\mathrm{T} 2$ sequences.

How does MR enteroclysis compare with WCE in the brave new world of imaging? This question was addressed in a prospective German study. ${ }^{43}$ Thirty six consecutive patients were referred for suspected Crohn's disease, obscure bleeding, and surveillance after small bowel tumours. Sixteen of 36 patients had documented Crohn's disease and two of 36 had suspected Crohn's disease. One patient suspected of Crohn's disease was ultimately found to have Clostridium difficile colitis. Therefore, 17 patients with probable or confirmed Crohn's disease were studied. None of the patients had used non-steroidal anti-inflammatory drugs. WCE demonstrated inflammatory lesions in the jejunum in five of 17 patients, and in the proximal ileum in seven of 17 patients. MRI found suspected lesions in the proximal and mid small bowel in only one patient. In the terminal ileum, both techniques found inflammatory changes in eight of 17 patients. MRI enteroclysis also detected extraluminal pathologies in two of 17 cases which were not detected by capsule. In this study, WCE was superior to MR enteroclysis in finding lesions in the jejunum and proximal ileum. There were no significant differences between WCE and MR enteroclysis in the detection of intestinal inflammation in the terminal ileum. The finding of additional lesions in the proximal small bowel on WCE however did not result in any changes to the therapeutic approach. MR enteroclysis was advantageous in imaging extraluminal pathology.

Hence MRI has proven to have a definitive role in imaging in Crohn's disease. Whether it should supplant routine barium imaging is still debatable, especially since the best method of conducting MRI (with or without enteroclysis),

\section{Magnetic resonance imaging}

- The main advantage of MRI over other modalities is its ability to differentiate active inflammation from fibrosis in a thickened bowel segment.

- MRI is safe in pregnancy and in renal failure.

- The use of non-ionising radiation makes MRI an attractive diagnostic test, particularly in young patients of reproductive age.

- Whether MRI should supplant routine barium imaging is still debatable, especially as the best way of conducting the study (with or without enteroclysis) remains to be proven. and what type of contrast optimises the study remain to be proved.

Figure 2 is an example of an MRI and SBFT in the same patient who had an intestinal fistula better demonstrated on MRI.

\section{MRI COLONOGRAPHY}

Is there a role for MRI in UC or colonic Crohn's disease? Certainly the gold standard in these entities is colonoscopy which not only gives an assessment of the mucosa but allows biopsy and therapeutic intervention. Yet colonoscopy is invasive, may involve patient discomfort, and carries with it a risk of perforation. MRI colonography is an evolving alternative and may have a role if endoscopy is incomplete, contraindicated or declined by the patient. MRI studies assessing the colon in patients with inflammatory bowel disease are few in number and compare clinical parameters such as the Crohn's disease clinical index or laboratory parameters such as $\mathrm{C}$ reactive protein. Madsen and colleagues $^{44}$ and Maccioni and colleagues ${ }^{45}$ demonstrated correlations between these parameters and MRI indicators of inflammation such as wall thickening and wall contrast uptake. Conversely, a study by Schunk et al did not find a correlation between laboratory findings and MRI findings. ${ }^{46}$ Recently, Schreyer et al have prospectively compared the performance of MRI colonography with conventional endoscopy, using bowel wall thickness and enhancement as MRI features of inflammation. ${ }^{47}$ The authors chose to study MRI rather than CT colonography, citing the benefit of lack of radiation in younger patients. Twenty two consecutive patients with known or suspected inflammatory bowel disease were enrolled. All patients underwent a colon preparation followed by MRI colonography with positive luminal contrast. Colonoscopy was performed on the same day. The colon, including the terminal ileum, was divided into seven segments for MRI assessment and correlation with endoscopy. Of the 154 total evaluated segments, MR colonography demonstrated a sensitivity and specificity of $58.8 \%$ and $91.4 \%$ in UC and $31.6 \%$ and $100 \%$ in patients with Crohn's disease, respectively. In two patients with documented severe UC, no abnormalities were found on MRI. This likely reflects the fact that UC is characterised by mucosal inflammation rather than by bowel wall thickening. These findings were similar to an earlier study by Koh and colleagues $^{41}$ who compared the findings of MRI with endoscopy or surgery and found a sensitivity of 59\% for identification of all bowel segments with known active inflammation. However, on a per patient basis, an overall sensitivity of $91 \%$ and specificity of $71 \%$ were achieved. Another study by Schreyer and colleagues ${ }^{48}$ applied the dark lumen technique and this resulted in improved sensitivity in the detection of colitis from $31.6 \%$ to $55.1 \%$ in Crohn's colitis disease, for segmental grading.

Ajaj and colleagues ${ }^{49}$ studied the diagnostic accuracy of MRI colonography in 15 normal controls and 23 patients with suspected inflammatory bowel disease of the colon. Three dimensional $\mathrm{Tl}$ weighted data sets were collected following rectal administration of water in association with intravenous gadolinium. The sensitivity and specificity of MR colonography was $87 \%$ and $100 \%$, respectively, compared with the gold standard of histopathological data from conventional colonoscopy.

Newer techniques in MRI such as the use of different contrast media are likely to improve the sensitivity of MRI 


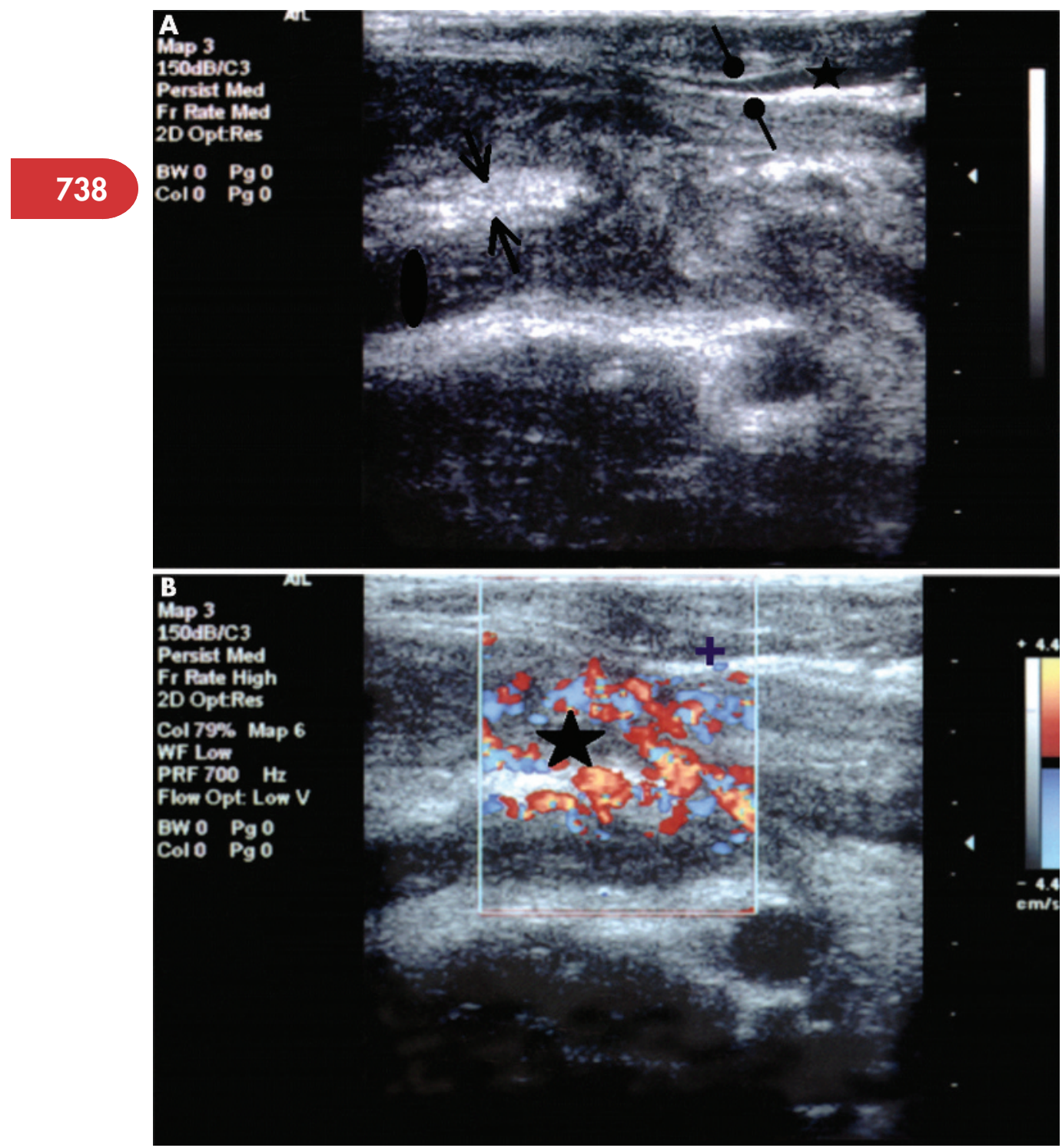

Figure 3 (A) Abdominal ultrasound in an adolescent with known ileal Crohn's disease and symptoms suggestive of partial small bowel obstruction. The echogram reveals a small bowel loop with a markedly thickened wall (14 mm, normal arrows, left side) in the right lower quadrant, corresponding to the non-terminal ileum. The bowel lumen (oval) is distended. A normal small bowel loop is illustrated in the right upper quadrant, with normal wall ( $3 \mathrm{~mm}$, round arrows) and a normal lumen (star). (B) High pulse abdominal Doppler ultrasound in an adolescent with known ileal Crohn's disease and symptoms suggestive of partial small bowel obstruction. The Doppler study reveals an area with greatly increased intramural mucosal blood flow, with $>5$ vessels $/ \mathrm{cm}^{2}$ of the area of thickened bowel wall (star). Vessel density is normal in an adjacent uninvolved ileal area $(+)$. These findings were compatible with an inflammatory nature to the obstructive symptoms, directing the patient to medical therapy, rather than a surgical intervention. These figures were kindly provided by $\mathrm{Dr}$ Ernest Seidman, McGill University, Montreal, Canada.

colonography in patients with Crohn's disease and UC. However, it is not likely to supplant colonoscopy in UC in particular. Whether or not it can be used to distinguish Crohn's colitis from UC or help solve indeterminate colitis remains to be studied.

\section{COMPUTED TOMOGRAPHY ENTEROCLYSIS}

CT scan has been an important tool in the diagnosis and follow up of inflammatory bowel disease. In 1987, Fishman et al reported that clinical decisions were altered in $28 \%$ of patients who had a CT scan. ${ }^{50}$ Like MRI, CT can depict segmental thickening, extraluminal lesions, and complications such as sinus tracts, fistulas, and abscesses. While CT and MRI both provide cross sectional imaging of extraintestinal organs, a significant disadvantage of CT is its radiation, which is greater than that of small bowel $x$ ray. MRI has also performed better than helical CT in the delineation of disease in 26 patients with known Crohn's disease ${ }^{51}$ and is superior to CT in the documentation and differentiation of fistulous tracts in the pelvis. ${ }^{52}$

Conventional CT is limited in its assessment of the small bowel because artefacts are produced by collapsed bowel loops and there is no distension of the small bowel as in conventional SBE. A relatively new diagnostic tool is CT enteroclysis which combines conventional small bowel enteroclysis with helical CT. ${ }^{1}$ The advantage offered by enteroclysis is distension of the small bowel and therefore intraluminal visualisation. The advantage of conventional CT is speed, resolution of volumetric data set, and optimal utilisation of the contrast media. ${ }^{1}$ The latter allows visualisation of extraintestinal manifestations of the disease. In 2002, Hassan and colleagues ${ }^{53}$ published an original prospective study comparing CT enteroclyis to ileocolonoscopy in patients with known or suspected Crohn's disease. Overall sensitivity of CT enteroclysis was $86.7 \%$ and specificity was $100 \%$ in patients without a previous ileal resection. CT enteroclyis, however, did not detect recurrence of disease in the neoterminal ileum in patients with prior surgery.

The disadvantage of CT scan compared with conventional barium studies is the lack of dynamic information; differentiation between peristalsis and skip lesions may be difficult. ${ }^{1}$ Compared with MRI, CT has greater availability, is less expensive, and is less time consuming. MRI, however, offers static and dynamic imaging capabilities. ${ }^{1}$ MRI does not 


\section{Ultrasound}

- In one study, US, using oral polyethylene glycol solution, had a sensitivity and specificity for Crohn's disease of $91 \%$ and $96 \%$, respectively.

- Doppler US is another emerging technology which can increase the sensitivity of transabdominal US and potentially may provide information about disease activity.

- The role of endoscopic US in inflammatory bowel disease is limited to perianal disease.

involve ionising radiation, has excellent soft tissue resolution, and is safe in renal failure and pregnancy.

\section{TRANSABDOMINAL ULTRASOUND}

Over the past two decades ultrasound (US) has been studied as an emerging modality to identify bowel wall thickening. Shortcomings include a high interobserver variation and lack of standardisation. Visibility is difficult in the obese. The presence of gas in the intestinal lumen does not allow thorough visualisation of the bowel. A meta-analysis of the role of US in the detection of Crohn's disease demonstrated US sensitivity and specificity in the detection of Crohn's disease as $75-94 \%$ and $67-100 \%$, respectively. ${ }^{54} \mathrm{New}$ developments in abdominal US include oral contrast enhanced bowel US and Doppler US. Parente et al conducted a study of 102 patients with proven Crohn's disease. ${ }^{55}$ All patients underwent abdominal US before and after ingestion of polyethylene glycol solution. They reported that the sensitivity of conventional and oral contrast US was $91.4 \%$ and $96.1 \%$, respectively. Sensitivity for detecting strictures was $74 \%$ in conventional US and $89 \%$ in the oral contrast study.

Doppler ultrasonography is based on the role of the vasculature in inflammatory states, including inflammatory bowel disease. Recently, Yekeler and colleagues ${ }^{56}$ demonstrated that evaluation of the superior mesenteric artery by Doppler in conjunction with evaluation of affected bowel

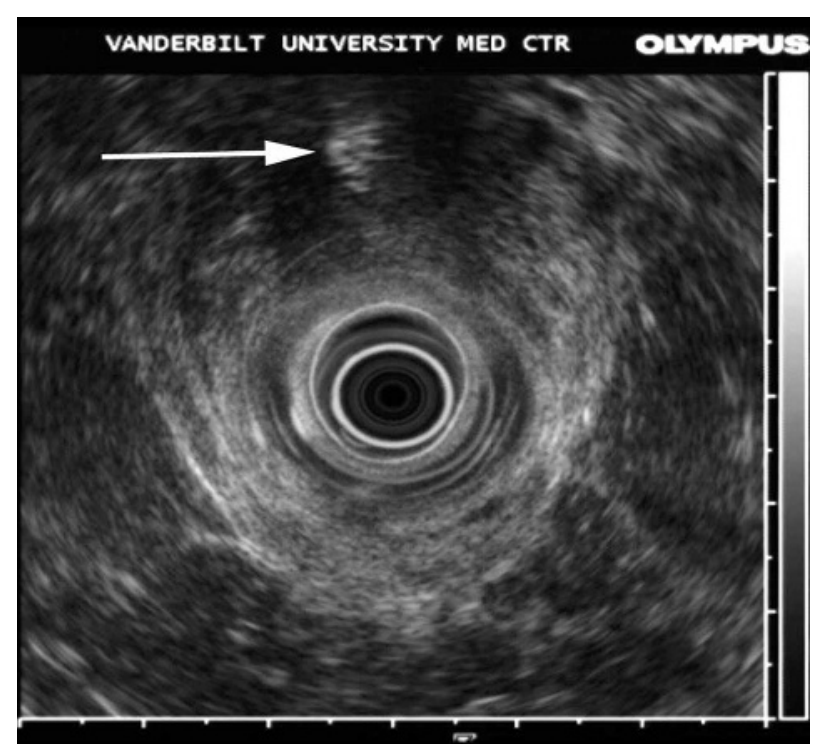

Figure 4 A perirectal fistula identified at rectal endoscopic ultrasound. The figure was kindly provided by Dr David Schwartz, Vanderbilt University, Nashville, Tennessee, USA. segments by gray scale is an excellent method of assessing disease activity. Sensitivity was $83 \%$ and specificity $100 \%$.

Figure 3 is an example of US, including with Doppler for diagnosing Crohn's disease.

\section{ENDOSCOPIC ULTRASOUND}

To date, the role of endoscopic US (EUS) in inflammatory disease is limited to perianal disease. A study by Schwartz and colleagues compared EUS, MRI, and examination under anaesthesia (EUA) in 34 patients with suspected Crohn's perianal fistulas. ${ }^{57}$ EUS, MRI, and EUA were comparable. The accuracy of all three modalities was 85\%: EUS 29/32 (91\% (95\% confidence interval (CI) 75-98)); MRI 26/30 (87\% (95\% CI 69-96\%)); and EUA 29/32 (91\% (95\% CI 75-98\%)). It was concluded that a combination of any two modalities was optimal.

A further study by Schwartz et al assessed whether the use of EUS as a guide to combination medical and surgical therapy during fistula healing led to a higher rate of resolution. ${ }^{58}$ Twenty one patients who presented with a perianal fistula were enrolled in the study. In 18 of 21 patients who presented with a fistula and received infliximab, ciprofloxacin and 6mercaptopurine, or azathioprine, the fistula stopped draining after a mean time of 10.6 weeks. Eleven of the 18 also had closure documented by EUS. Of these patients, seven have remained off infliximab and ciprofloxacin. The authors concluded that EUS may identify patients who can discontinue infliximab without the recurrence of a fistula.

Figure 4 is an example of an EUS identifying a perineal fistula arising from the rectum.

\section{DOUBLE BALLOON ENTEROSCOPY}

Endoscopic examination of the entire small bowel is technically very difficult. Push endoscopy is often possible only to the proximal jejunum. While WCE represents a monumental advance in the diagnosis of small bowel lesions, biopsies and endoscopic intervention are not possible. In 2001, Yamamoto and Kito developed the double balloon method as an insertion technique for the diagnosis and treatment of small bowel disorders. ${ }^{59}$ The double balloon which consists of an endoscope with a balloon at its end and an overtube with another balloon avoids the problem of stretching or looping of the small bowel. By shortening and simplifying the inserted small intestine onto the overtube, the endoscope is advanced distally. The tube may be inserted orally or rectally. Yamamoto and Kito reported that the entire small bowel was observed in $86 \%$ of cases attempted. Initially studied in cases of obscure bleeding, bleeding sites were identified in $76 \%$ of patients, which is comparable with the yield of WCE for this indication. ${ }^{60}$ In the largest series of 178 patients, Yamamoto and Kito reported two complications of small bowel perforation. ${ }^{59}$

DDW 2005 featured at least 16 presentations on double balloon enteroscopy, one of which dealt exclusively with Crohn's disease. ${ }^{61}$ Maeda et al performed double balloon endoscopy in 17 patients with Crohn's disease. A double balloon enteroscope was used antegradely in one patient and retrogradely in 16. Typical findings of Crohn's disease including longitudinal ulcers and a cobblestone appearance, inflammatory polyps, and erosions were observed in 13 patients. Biopsy with histological confirmation was obtained in 12 patients. Crohn's disease lesions could not be demonstrated in four patients given adhesions which made the procedure difficult. Indeed, the procedure should be approached with caution in patients with prior small bowel 

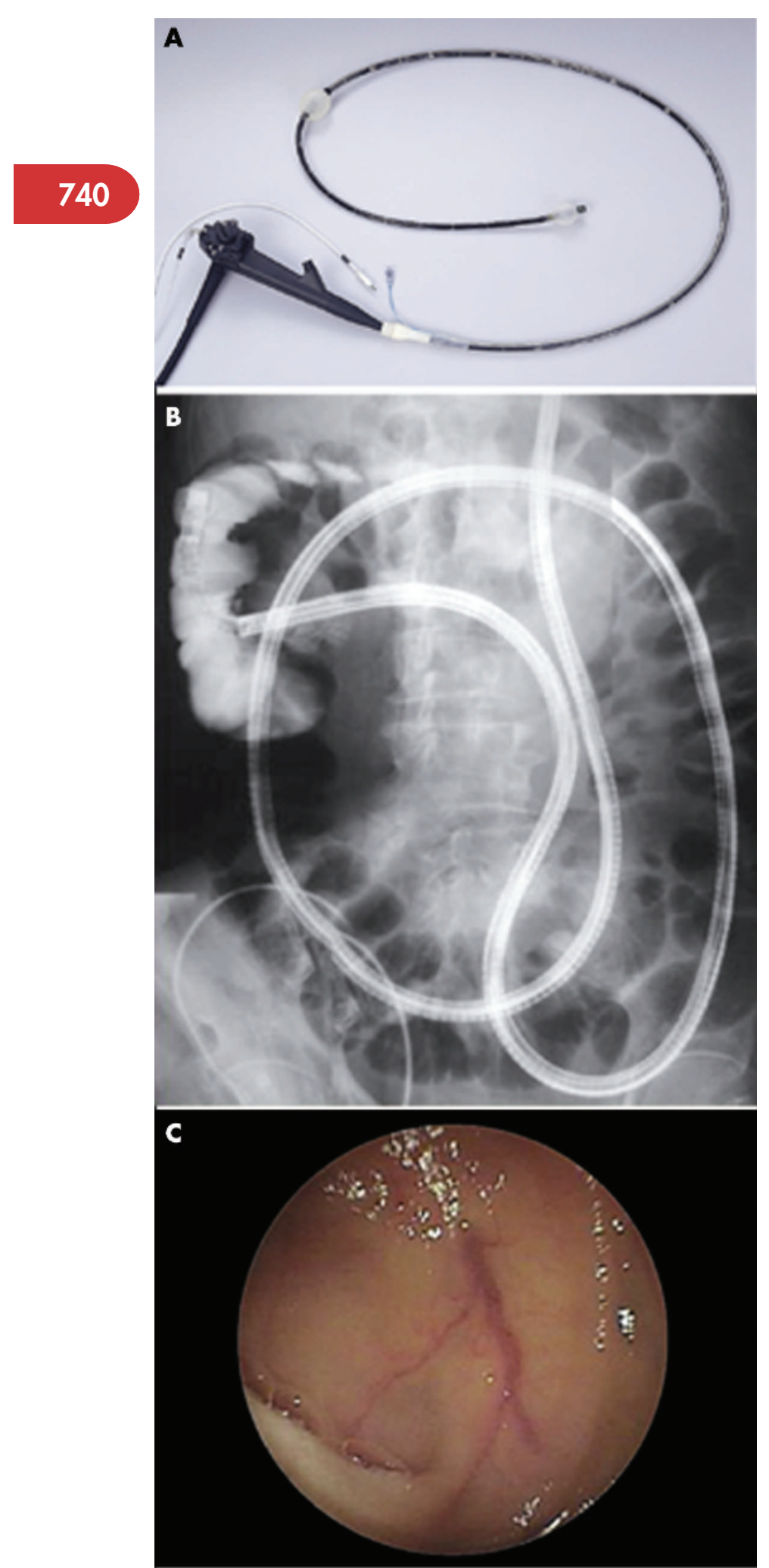

Figure 5 (A) Double balloon enteroscope. (B) Plain $x$ ray image of the double balloon enteroscope fully inserted per os, in the small bowel to the ileocaecal valve. (C) Small bowel image as viewed by double balloon enteroscope.

resections. ${ }^{61}$ The disadvantage of this procedure is patient discomfort and the laborious and time consuming nature of the modality. Lo and Leighton ${ }^{62}$ in the USA reported a mean procedure time of 115 minutes; Heine et al from Holland reported 82 minutes. ${ }^{63}$

Figure 5 shows the double balloon enteroscope, the appearance at fluoroscopy of the endoscope fully inserted into the small bowel per os, and a small bowel image, as viewed from the scope.

\section{SUMMARY}

Novel diagnostic advances in inflammatory bowel disease are complementary to clinical assessment, colonoscopy, and small bowel $x$ ray. The wireless video capsule, in its current form, is of value in the diagnosis of Crohn's disease when the condition is suspected and conventional studies are negative or technically impossible. The sensitivity of WCE is very high but specificity has yet to be determined. MRI, with or without enteroclysis, has a well established role. It uses non-ionising radiation and therefore is a potentially ideal imaging modality, especially in patients who require repeated studies. MRI is advantageous in patients with equivocal studies and is a crucial tool in distinguishing inflammation from fibrosis in patients with obstructive symptoms. Endoscopic US has a role primarily in the assessment of perianal disease. The roles of MR colonography, Doppler US, and double balloon enteroscopy hold promise but further studies are necessary as their roles are not well defined. Inflammatory bowel disease is a clinical diagnosis aided by imaging studies. Conventional imaging studies remain the mainstays of investigation but novel imaging techniques developed and enhanced over the past five years have greatly advanced diagnostic capabilities.

\section{ACKNOWLEDGEMENTS}

Dr Charles Bernstein is supported in part by a Canadian Institutes of Health Research Investigator Award and by a Crohn's and Colitis Foundation of Canada Research Scientist Award.

\section{Authors' affiliations}

B A MacKalski, C N Bernstein, University of Manitoba inflammatory Bowel Disease Clinical and Research Centre and University of Manitoba Department of Internal Medicine, Winnipeg, Manitoba, Canada Conflict of interest: None declared.

\section{REFERENCES}

1 Turetsschek K, Gasche C. Current status and new imaging trends in IBD. In: Bernstein CN, eds. The Inflammatory Bowel Disease Yearbook. London: Remedica Publishing, 2003:129-45.

2 Marshall JB, Barthel JS. The frequency of total colonoscopy and terminal ileal intubation in the 1990s. Gastrointest Endosc 1993;39:518-20.

3 Harewood GC, Mattek NC, Holub JL, et al. Variation in practice of ileal intubation among diverse endoscopy settings: results from a national endoscopic database. Aliment Pharmacol Ther 2005;22:571-8.

4 Wills JS, Lobis IF, Dentstman FJ. Crohn's disease. State-of-the-Art. AJR Am J Roentgenol, 1997;202:597-610.

5 Schreyer AG, Golder S, Seitz J, et al. New diagnostic avenues in inflammatory bowel disease. Dig Dis 2003;21:129-37.

6 Ott DJ, Chen YM, Gelfand DW, et al. Detailed per oral small bowel exam vs. enteroclysis. II. Radiographic accuracy. Radiology 1985;7:121-5.

7 Maglinte DDT, Chernish SM, Kelvin FM, et al. Crohn's disease of the small intestine: accuracy and relevance of enteroclysis. Radiology 1992; 184:541-5.

8 Herlinger H, Caroline DF. Crohn's disease. In: Gore RM, Levine MS, Laufer I, eds. Textbook gastrointestinal radiology. Philadelphia, Pa: Saunders, 1994:824-44.

9 Bernstein CN, Boult IF, Greenberg HM, et al. A prospective randomized comparison between small bowel enteroclysis and small bowel follow through in Crohn's disease. Gastroenterology 1997;113:390-8.

10 Munkholm P. Crohn's disease-occurrence, course, and prognosis in epidemiologic cohort study. Dan Med Bull 1997:44:287-302.

11 Hara A, Leighton J, Sharma V, et al. Comparison of capsule endoscopy, standard endoscopy, barium examination, and CT. Radiographics 2005;25:698-711. 
12 Hara AK. Capsule endoscopy: the end of the barium small bowel examination? Abdom Imaging 2005;30:179-83.

13 Costamagna G, Shah SK, Riccioni ME, et al. A prospective trial comparing small bowel radiographs and video capsule endoscopy for small bowel disease. Gastroenterology 2002;123:999-1005.

14 Fireman Z, Mahajana E, Broide E, et al. Diagnosing small bowel Crohn's disease with wireless capsule endoscopy. Gut 2003;42:390-2.

15 Legnani $\mathbf{P}$, Kornbluth A. Video capsule endoscopy in inflammatory bowel disease 2005. Curr Opin Gastroenterol 2005;21:438-42.

$16 \mathrm{Ge} \mathrm{ZZ,} \mathrm{Hu} \mathrm{YB,} \mathrm{Xiao} \mathrm{SD.} \mathrm{Capsule} \mathrm{endoscopy} \mathrm{in} \mathrm{the} \mathrm{diagnosis} \mathrm{of} \mathrm{small} \mathrm{bowe}$ Crohn's disease. World J Gastroenterol 2004; 10:1349-52.

17 Herrerias JM, Caunedo A, Rodriguez-Tellez M, et al. Capsule endoscopy in patients with suspected Crohn's disease and negative endoscopy. Endoscopy 2003:35:564-8.

18 Mow WS, Lo SK, Targan SR, et al. Initial experience with wireless capsule endoscopy in the diagnosis and management of inflammatory bowel disease. Clin Gastroenterol Hepatol 2004;2:31-40.

19 Liangpunsakul S, Maglinte D, Rex DK. Comparison of wireless capsule endoscopy and conventional radiologic methods in the diagnosis of small bowel disease. Gastrointest Endosc Clin N Am 2004; 14:43-50.

20 Voderholzer WA, Ortner M, Rogalla P, et al. Diagnostic yield of capsule endoscopy in comparison with computed tomography enteroclysis. Endoscopy 2003;35:1009-14.

21 Voderholzer WA, Beinhoelzl J, Rogalla P, et al. Small bowel involvement in Crohn's disease: a prospective comparison of wireless capsule endoscopy and computed tomography enteroclysis. Gut 2005;54:369-73.

22 Eliakim R, Fischer D, Suissa A, et al. Wireless capsule endoscopy is a superior diagnostic tool in comparison to barium follow-through and computerized tomography in patients with suspected Crohn's disease. Eur J Gastroenterol Hepatol 2003; 15:363-7.

23 Eliakim R, Suissa A, Yassin K, et al. Wireless capsule endoscopy compared to barium follow through and computerized tomography in patients with suspected Crohn's disease, final report. Dig Liver Dis 2004;36:519-22.

24 Arguelles-Arias F, Caunedo A, Romero J. The value of capsule endoscopy in pediatric patients with suspected Crohn's disease. Endoscopy 2004;36:869-73

25 Barkay O, Moshkowitz M, Reif S. Crohn's disease diagnosed by wireless capsule endoscopic findings in adolescents with abdominal pain, proteinlosing enteropathy, anemia and negative endoscopic and radiologic findings. Isr Med Assoc J 2005; 7:262-3

26 Maiden L, Thiodleisson B, Theodors A, et al. A quantitative analysis of NSAID induced small bowel pathology by capsule endoscopy. Gastroenterology 2005; 128:1172-8.

27 Graham DY, Opekun AR, Willingham FF, et al. Visible small intestinal mucosal injury in chronic NSAID users. Clin Gastroenterol Hepatol 2005;3:55-9.

28 Barkin J, Friedman S. Wireless capsule endoscopy requiring surgical intervention: the world's experience. Gastroenterol Endosc. 2002;55: S907 (abstract)).

29 Kornbluth A, Legnani P, Lewis B. Video capsule endoscopy in inflammatory bowel disease: past, present and future. Inflamm Bowel Dis 2004;10:278-85.

30 Boivin ML, Lochs H, Voderholzer WA. Does passage of a patency capsule indicate small bowel patency? A prospective trial? Endoscopy 2005;37:808-15.

31 Koebel G, Schmiedl U, Majer MC, et al. Diagnosis of fistulae and sinus tracts in patients with Crohn's disease. Value of MR imaging. AJR Am J Roentgenol, 1989;152:999-1003

32 Haggett PJ, Moore NR, Shearman JD, et al. Pelvic and perineal complications of Crohn's disease: Assessment using magnetic resonance imaging. Gut 1995;36:407-10.

33 Yousem DM, Fishman EK, Jones B. Crohn's disease; perirectal and perianal findings at CT. Radiology 1988;167:331-4.

34 Laghi A, Borrelli O, Paolantonio P, et al. Contrast enhanced magnetic resonance imaging of the terminal ileum in children with Crohn's disease. Gut 2003;52:393-7.

35 Schreyer AG, Geissler AG, Albrich H, et al. Abdominal MRI after enteroclysis or with oral contrast in patients with suspected or proven Crohn's disease. Clin Gastroenterol Hepatol 2004;2:491-7.

36 Rieber A, Wruk D, Potthast S, et al. Diagnostic imaging in Crohn's disease: comparison of magnetic resonance imaging and conventional techniques. Int J Colorectal Dis 2000;15:176-81.

37 Ochsenkuhn T, Herrmann K, Schoenberg SO, et al. Crohn's disease of the small bowel proximal to the terminal ileum: detection by MR-enteroclysis. Scand J Gastroenterol 2004;39:953-60.
38 Holzknebcht N, Helmberger T, von Ritter $\mathrm{C}$, et al. MRI of the small intestine with rapid sequences in Crohn's disease after enteroclysis with oral iron particles. Radiologie 1998;38:29-36.

39 Bernstein CN, Greenberg H, Boult I, et al. A prospective comparison of MRI versus small bowel follow through in recurrent Crohn's disease. Am J Gastroenterol 2005; 100:2493-502.

40 Masselli G, Brizi GM, Parrella, et al. Crohn disease: magnetic resonance enteroclysis. Abdom Imaging 2004;29:326-34.

41 Koh DM, Miao Y, Chinn RJ, et al. MR imaging evaluation of the activity of Crohn's disease. AJR Am J Roentgenol 2001;177:1325-32.

42 Ebinger M, Rieber A, Liedl R. Cost-effectiveness of magnetic resonance imaging and enteroclysis in the diagnosis of Crohn's disease. Intl J Technol Assess Health Care 2002;18:711-17.

43 Golder SK, Schreyer AG, Endlicher E, et al. Comparison of capsule endoscopy and magnetic resonance (MR) enteroclysis in suspected small bowel disease. Int J of Colorectal Dis 2006;21:97-104.

44 Madsen SM, Thomsen HS, Munkholm P, et al. Inflammatory bowel disease evaluated by low-field magnetic resonance imaging. Comparison with endoscopy, 99m Tc-HMPAO leukocyte scintigraphy, conventional radiography and surgery. Scand J Gastroenterol 2002;37:307-16.

45 Maccioni F, Viscido A, Broglia L, et al. Evaluation of Crohn's disease activity with MRI. Abdom Imaging 2000;25:219-28.

46 Schunk K, Kern A, Oberhlzer K, et al. Hydro-MRI in Crohn's disease: appraisal of disease activity. Invest Radiol 2000;35:431-7.

47 Schreyer AG, Rath HC, Kikinis R, et al. Comparison of magnetic resonance imaging colonoscopy for the assessment of intestinal inflammation in patients with inflammatory bowel disease: a feasibility study. Gut 2005;54:250-6.

48 Schreyer AG, Golder S, Sceibl K, et al. Dark lumen magnetic resonance enteroclysis in combination with MRI colonography for whole bowel assessment in patients with Crohn's disease: first clinical experience. Inflamm Bowel Dis 2005; 11:388-94

49 Ajaj W, Pelster G, Treichel U, et al. Dark lumen magnetic resonance colonography: comparison with conventional colonoscopy for detection for colorectal pathology. Gut 2003;52:1738-43.

50 Fishman EK, Wolf EJ, Jones B, et al. CT evaluation of Crohn's disease: effect on patient management. AJR Am J Roentgenol, 1987;148:537-40.

51 Low RN, Francis IR, Politiske D, et al. Crohn's disease evaluation: comparison of contrast-enhanced MR imaging and single-phase helical CT scanning. $J$ Magn Reson Imaging 2000;1 1:127-35.

52 Morris J, Spencer JA, Ambrose NS. MR imaging classification of perianal fistulas and its implications for patient management. Radiographics 2000;20:623-635

53 Hassan C, Cerro P, Zullo A, et al. Computed tomography enteroclysis in comparison with ileoscopy in patients with Crohn's disease. Int J Colorectal Dis 2003;18:121-5.

54 Fraquelli M, Colli A, Casazza G, et al. Role of US in detection of Crohn's disease: a meta-analysis. Radiology 2005;236:95-101.

55 Parente F, Greco S, Molteni $M$, et al. Oral contrast enhanced bowe ultrasonography in the assessment of small intestinal Crohn's disease. A prospective comparison with conventional ultrasound, $\mathrm{x}$-ray studies and ileocolonoscopy. Gut 2004:53:1652-7.

56 Yekeler E, Danalioglu A, Movaseghi B, et al. Crohn disease activity evaluated by Doppler ultrasound of the superior mesenteric artery and the affected small bowel segments. J Ultrasound Med 2005;24:59-65.

57 Schwartz DA, Wiersema M, Dudiak KM, et al. A comparison of endoscopic ultrasound, magnetic resonance imaging, and exam under anesthesia for evaluation of Crohn's perianal fistulas. Gastroenterology 2001;121:1064-72.

58 Schwartz DA, White CM, Paul E, et al. Crohn's per anal fistulas. Inflamm Bowel Dis 2005;11:727-32.

59 Yamamoto H, Kito H. Double-balloon endoscopy. Curr Opin Gastroenterol 2005;21:573-7.

60 May A, Nachbar L, Wardak A, et al. Double-balloon enteroscopy. Preliminary experience in patients with obscure gastrointestinal bleeding or chronic abdominal pain. Endoscopy 2003;35:985-91.

61 Maeda O, Ando T, Watanabe R, et al. Usefulness of double-balloon enteroscopy in Crohn's disease. Gastroenterology. 2005; 128: A315 (abstract)).

62 Lo SK, Leighton JA, Ross A, et al. Double balloon push enteroscopy: Technical details and early experience in 6 US tertiary care centers. Gastroenterology. 2005; 128: M1365 (abstract)).

63 Heine DG, Hadithi M, Groenen EJ, et al. Double balloon enteroscopy: the Dutch one year experience indication, yield, and complications in a series of 125 cases. Gastroenterology. 2005;128: M1334 (abstract)). 